\title{
Unerwünschte Ereignisse durch unsachgemäß durchgeführte Psychotherapie
}

\author{
Eine Pilotstudie zum Inventar zur Erfassung von Kunstfehlern und Kunstfehlerfolgen \\ in der Psychotherapie (IKKIP)
}

Unwanted Events Caused by Incorrectly Conducted Psychotherapy

\author{
A Pilot Study of the Inventory for the Assessment of Malpractice and its \\ Consequences in Psychotherapy
}

Autoren

Romina Gawlytta ${ }^{1,}$, Dominique Schwartze ${ }^{1,{ }^{*}}$, Désirée Schönherr ${ }^{1}$, Andrea Schleu ${ }^{2}$, Bernhard Strauß ${ }^{1}$

Institute

1 Institut für Psychosoziale Medizin und Psychotherapie, Universitätsklinikum Jena

2 Verein Ethik in der Psychotherapie, Essen

Schlüsselwörter

Psychotherapieforschung, Kunstfehler, Grenzverletzungen, Nebenwirkungen, Fragebogen

Key words

psychotherapy research, malpractice, boundary violations, side effects, questionnaire

Bibliografie

DOI https://doi.org/10.1055/a-1026-1577

Psychiat Prax 2019; 46: 460-467

(c) Georg Thieme Verlag KG Stuttgart · New York ISSN 0303-4259

Korrespondenzadresse

Romina Gawlytta, M.Sc. Psych., Universitätsklinikum Jena, Institut für Psychosoziale Medizin und Psychotherapie,

Stoystraße 3, 07740 Jena

romina.gawlytta@med.uni-jena.de

\section{ZUSAMMENFASSUNG}

Ziel der Studie In einer Pilotstudie wurde das neu entwickelte Inventar zur Erfassung von Kunstfehlern und Kunstfehlerfolgen in der Psychotherapie (IKKIP) erstmals eingesetzt, um die Häufigkeit therapeutischen Fehlverhaltens aus Patientensicht zu erfassen, die resultierende Belastung zu erheben und Prädiktoren zu identifizieren.

Methodik Ausgewertet wurden Daten von N=165 Patienten mit Psychotherapieerfahrung, die an der Online-Befragung teilnahmen.

\footnotetext{
* Diese Publikation wurde in geteilter Erstautorenschaft erstellt.
}

Ergebnisse Die Patienten berichteten im Mittel $M=16,21$ Ereignisse, die im Sinne von Kunstfehlern interpretierbar sind, und zu einer Belastung mittelmäßiger Schwere führten. Während Ereignisse der Kategorie „Mangelhafte Diagnostik/Technikanwendung“ am häufigsten berichtet wurden, gingen sexuelle Grenzverletzungen mit dem höchsten Belastungsgrad einher.

Schlussfolgerung Die Ergebnisse belegen die Notwendigkeit einer differenzierten und systematischen Erfassung von Kunstfehlern und deren Folgen. Das IKKIP scheint dabei vor allem für den Forschungsbereich geeignet.

\section{ABSTRACT}

Objective A pilot study was conducted to test a newly developed inventory for the assessment of malpractice in psychotherapy and its consequences. We aimed to examine the frequency of boundary crossings and violations in the context of psychotherapy from a patient's perspective and the resulting burden as well as to identify potential predictors.

Methods Data assessment was realized in an online-based survey. The final sample existed of $N=165$ patients who underwent prior psychotherapeutic treatment.

Results Patients reported $M=16.21$ events which can be interpreted in terms of malpractice resulting in a mediocre level of distress. While malpractice summarized as "inadequate use of diagnostics/techniques" was reported most frequently, sexual boundary violations were associated with the highest levels of distress.

Conclusion The results demonstrate the need of a differential and systematic assessment of malpractice in psychotherapy. The new inventory especially seems to be suited for research purposes. 


\section{Einleitung}

In den vergangenen Jahrzehnten wurde im Rahmen der Psychotherapieforschung primär die Frage nach der Wirksamkeit psychotherapeutischer Behandlungen intensiv beforscht [1]. Dass Psychotherapie bei einer Vielzahl verschiedener psychischer Störungen indiziert und wirksam ist, ist durch wissenschaftliche Forschungsergebnisse entsprechend gut belegt. Inwiefern eine psychotherapeutische Behandlung auch mit Risiken und Nebenwirkungen einhergeht oder aus einer Psychotherapie negative Effekte für Patienten ${ }^{1}$ resultieren können und wodurch diese bedingt sind, wurde weniger intensiv betrachtet, weshalb bis dato eine vergleichsweise geringe Zahl an Forschungsbefunden dazu vorliegt.

Ein Grund dafür ist der lange Zeit fehlende Konsens darüber, wie negative und unerwünschte Effekte von Psychotherapie überhaupt definiert werden können und wie in diesem Zusammenhang zwischen verschiedenen Begrifflichkeiten (z. B. Risiken, Nebenwirkungen, Kunstfehlerfolgen, Misserfolge, Nonresponse) differenziert werden muss. Erst in den letzten Jahren gibt es zunehmend Bemühungen, diese Lücke in der Psychotherapieforschung zu schließen.

Im Hinblick auf eine einheitliche Definition wird im deutschsprachigen Raum im Bereich der Forschung zu unerwünschten Entwicklungen und negativen Effekten von Psychotherapie inzwischen häufig dem Systematisierungsvorschlag von Haupt und Kollegen [2] gefolgt. Dabei wird zunächst differenziert betrachtet, ob unerwünschte Ereignisse (UE) durch eine Psychotherapie verursacht worden sind, also als negative Therapiewirkungen bezeichnet werden können, oder unabhängig von der Behandlung auftreten, beispielsweise bedingt durch den natürlichen Krankheitsverlauf oder äußere Ereignisse. Im Falle negativer Therapiewirkungen ist weiterführend abzuklären, inwiefern diese möglicherweise durch eine inkorrekt durchgeführte Behandlung (z. B. Fehler in der Indikationsstellung, Verfahrensfehler, Grenzüberschreitungen [GrÜ] und/oder -verletzungen [GrV] durch den Therapeuten) entstanden sind und somit als sogenannte Kunstfehlerfolgen bezeichnet werden können. Wenn UE nicht auf therapeutisches Fehlverhalten zurückzuführen sind, sondern trotz einer korrekt durchgeführten Behandlung auftreten, wird der Begriff der Nebenwirkungen verwendet. In der Arbeit von Linden und Kollegen werden angelehnt an dieses Modell Entscheidungsschritte postuliert, die im Prozess der Erfassung und Einordnung von UE hilfreich sind [3].

Da diese Systematik jedoch noch nicht lange etabliert ist, wurde das Auftreten von UE in bisherigen Studien (z.B. [4]) meist erfasst, ohne spezifische Aussagen darüber zu treffen, ob die negativen Effekte auf Kunstfehler zurückzuführen sind, als Nebenwirkungen zu interpretieren sind oder keinerlei Bezug zur Therapie hergestellt werden kann.

Ein wesentlicher Grund dafür ist, dass bislang nur wenige Inventare existieren, die entsprechend der o.g. Systematisierung hinsichtlich der Ursachen von UE im Rahmen von Psychothera-

\footnotetext{
${ }^{1}$ Im Sinne der besseren Lesbarkeit wird in diesem Artikel von Patienten und Therapeuten gesprochen, gemeint sind damit jedoch stets auch Patientinnen und Therapeutinnen.
}

pie unterscheiden. Bei den meisten der gegenwärtig existierenden Fragebögen, wie beispielsweise dem Negative Effects Questionnaire (NEQ) [5], erfolgt die Bearbeitung in zwei Schritten. Dabei sollen die Befragten zunächst angeben, ob ein UE (z. B. „Ich fühlte mich gestresster.“) aufgetreten ist oder nicht. Im Falle des Auftretens ist danach zu evaluieren, ob dieses UE aus Sicht der Befragten auf die Psychotherapie oder auf andere Umstände zurückgeführt wird. Durch dieses Vorgehen ist es zwar möglich, die subjektive Sicht der Befragten bezüglich eines kausalen Zusammenhangs zwischen dem UE und der Psychotherapie zu erfassen. Auf dieser Grundlage kann jedoch keine Aussage darüber getroffen werden, ob die Therapie sachgemäß im Sinne der Behandlungsintegrität durchgeführt wurde (vgl. [6]) und der Therapeut ethisch korrekt gehandelt hat, womit noch immer Unklarheit darüber besteht, ob das UE als $\mathrm{Ne}$ benwirkung oder als Kunstfehlerfolge zu klassifizieren ist.

Mit dem Inventar zur Erfassung negativer Effekte in der Psychotherapie (INEP) [7] wird neben möglichen Folgen einer psychotherapeutischen Behandlung auch das Auftreten einiger Kunstfehler erfasst (z. B. „Mein/e Therapeut/in zwang mich Dinge zu tun [Konfrontationen, Rollenspiele etc.], die ich eigentlich gar nicht wollte."). Jedoch wird durch die INEP-Items nur ein geringer Teil möglicher therapeutischer Fehlverhaltensweisen abgedeckt.

Dass die Notwendigkeit einer möglichst breiten, systematischen Erfassung von Kunstfehlern und deren Folgen besteht, belegen zum einen Ergebnisse aus qualitativen Inhaltsanalysen von Beschwerdebriefen an die Psychotherapeutenkammern, in denen Patienten über das Auftreten von Kunstfehler und unethisches Verhalten im Rahmen ihrer Therapie berichten sowie auf daraus resultierende negative Konsequenzen aufmerksam machen (z.B. [8]). Zum anderen zeigen die Ergebnisse der Arbeit des sog. Ethikvereins e. V. - Ethik in der Psychotherapie die Wichtigkeit einer systematischen wissenschaftlichen Auseinandersetzung mit der Thematik der Kunstfehler und deren Folgen auf [9-12]. Der seit 2004 bestehende, ehrenamtlich arbeitende Verein ist eine gemeinnützige Einrichtung, an die sich Patienten, Therapeuten, Institutionen und Auszubildende kostenlos und anonym wenden können, wenn der Bedarf nach niederschwelliger Beratung zur Klärung ethischer Fragen in der Psychotherapie besteht. Auf Basis der vom Ethikverein e. V. dokumentierten Fälle und Daten wurde ein Kategoriensystem für therapeutisches Fehlverhalten entwickelt $[13,14]$, mithilfe dessen Kunstfehler innerhalb 12 verschiedener Kategorien klassifiziert werden können: Sexuelle GrV; Soziale GrV; Befangenheit; Verletzung therapeutischer Basisvariablen; Mangelhafte Diagnostik/Technikanwendung; Verstoß gegen Informations-, Dokumentations- und Schweigepflicht; Ökonomischer/finanzieller Missbrauch; Therapieabbruch durch den Therapeuten; Mangelhafte Rahmenbedingungen; Sonstiges; GrV in der stationären Therapie; GrV in der Gruppentherapie.

Bislang existierte noch kein Inventar, das systematisch das Auftreten von Kunstfehlern und deren Folgen aus Patientensicht erfasst und durch das Kunstfehlerfolgen hinreichend von Nebenwirkungen abgegrenzt sowie in ihrem Schweregrad eingeschätzt werden können. 
Ausgehend vom vorgestellten Kategoriensystem wurde daher ein neuer Fragebogen entwickelt, der diese Lücke schließen soll [15]. Anhand der vom Ethikverein e. V. dokumentierten Beschwerdeschilderungen sowie durch Literaturrecherchen zu bereits existierenden Fragebögen wurde ein vorläufiger Itempool entwickelt, welcher von drei klinischen Experten hinsichtlich Relevanz, Plausibilität und Verständlichkeit geprüft und folglich überarbeitet wurde. Mit dem resultierenden Inventar zur Erfassung von Kunstfehlern und Kunstfehlerfolgen in der Psychotherapie (IKKIP) können verschiedene Formen und Aspekte therapeutischen Fehlverhaltens (Technikfehler, GrÜ und GrV/ Missbrauch) entsprechend der zwölf oben genannten Beschwerdekategorien erfasst werden.

Das Inventar bildet darüber hinaus auch das Belastungserleben der Patienten infolge der aufgetretenen Kunstfehler ab. Letzteres wird als wichtig erachtet, da davon ausgegangen werden kann, dass unterschiedliche Verhaltensweisen eines Therapeuten nicht von allen Patienten gleichermaßen als problematisch wahrgenommen werden und das resultierende Belastungserleben entsprechend individuell sehr unterschiedlich sein kann.

Ziel der vorliegenden Pilotierungsstudie war es, den Einsatz des IKKIP erstmals in einer Stichprobe von Patienten mit Psychotherapieerfahrung zu erproben. Neben dem Ziel der Weiterentwicklung des Fragebogens ging es zudem vor allem darum, gezielt und systematisch das Auftreten grenzverletzender sowie grenzüberschreitender therapeutischer Situationen im Sinne von Kunstfehlern zu erfassen und zudem zu erheben, wie belastet Patienten durch deren Auftreten sind.

\section{Methodik}

\section{Fragebogen}

Das IKKIP umfasst 84 Items aus 12 Beschwerdekategorien, durch die das Auftreten von Kunstfehlern im Rahmen einer psychotherapeutischen Behandlung retrospektiv aus Patientensicht abgefragt wird. Dabei schwankt die Anzahl der Items je Kategorie zwischen 3 (Kategorie „Befangenheit“) und 10 (Kategorie „Verletzung therapeutischer Basisvariablen“).

Die Bearbeitung des Fragebogens erfolgt gestuft in zwei Schritten. Zunächst ist die befragte Person dazu aufgefordert anzugeben, ob ein mögliches therapeutisches Fehlverhalten im Rahmen einer früheren oder einer gegenwärtig laufenden Psychotherapie aufgetreten ist oder nicht (Antwortformat „ja“/,nein“). Wenn diese Frage mit „ja“ beantwortet wird, soll im nächsten Schritt auf einer 5-stufigen Skala angeben werden, inwiefern sich die Person durch dieses Ereignis belastet gefühlt hat oder noch immer fühlt (von $0=$ „gar nicht“ bis 4 = „sehr stark").

Zusätzlich enthält das IKKIP Fragen mit offenem Antwortformat, für den Fall, dass durch die aktuelle Fragebogenversion nicht das gesamte Spektrum potenziell belastender Situationen abgedeckt wird.

\section{Erhebung und Analysen}

Die Studie wurde im Vorfeld bei der Ethikkommission der Universität Jena angezeigt (5425-02/18) und von dieser befürwor- tet. Im Zeitraum von Mai bis Juni 2018 fand die Datenerhebung im Rahmen einer Internet-Befragung über die Online-UmfrageApplikation LimeSurvey statt. Die Verbreitung der Informationen über die Studie und des Links zur Befragung erfolgte über Flyer in psychotherapeutischen Praxen und Ambulanzen sowie deutschlandweit in Foren und Selbsthilfegruppen für Menschen mit psychischen Störungen. Die Studienteilnehmer erhielten keine finanzielle Aufwandsentschädigung. Die Teilnahme erfolgte freiwillig und anonym. Zusätzlich zum IKKIP wurden auch demografische sowie klinische (z. B. Diagnose) Angaben des Patienten erfasst. Weiterhin waren die Teilnehmer aufgefordert, Angaben zum Therapeuten (z. B. Geschlecht, Berufsgruppe) sowie zur Psychotherapie (z.B. Behandlungssetting, -modalität, Therapieschule) zu machen.

In die finale Analyse, die mit dem Programm IBM SPSS 23.0 durchgeführt wurde, konnten die Daten von 165 Teilnehmern eingeschlossen werden. Aufgrund umfassend unvollständiger Angaben wurden 127 Datensätze von der Analyse ausgeschlossen.

Um zu untersuchen, ob demografische (z. B. Geschlecht Patient) oder klinische (z. B. Diagnose) Variablen die Anzahl der Kunstfehler oder der resultierenden Belastung vorhersagen können, wurden lineare Regressionen berechnet. Als Kriterium wurde die Anzahl der Kunstfehler oder der Grad der Belastung verwendet. In separaten Regressionen wurden die Prädiktoren Geschlecht des Patienten, Anzahl der Diagnosen, Geschlecht des Therapeuten, Beruf des Therapeuten, die Therapieschule und verschiedene Störungsbilder (Depression, Angst, Persönlichkeitsstörungen [PKS], Essstörungen, Traumafolgestörungen, Schizophrenie) untersucht.

\section{Ergebnisse}

\section{Stichprobenbeschreibung}

$N=129(78,2 \%)$ der Studienteilnehmer waren weiblich. Das Alter der Teilnehmer lag zwischen 16 und 75 Jahren $(M=40,5$ Jahre; $S D=13,8$ Jahre). Bezüglich des höchsten erreichten Bildungsabschlusses machten 7 Personen (4,2\%) keine Angaben, 2 (1,2\%) Teilnehmer berichteten, keinen Schulabschluss zu haben, 3 (1,8\%) hatten einen Hauptschulabschluss, 32 $(19,4 \%)$ die mittlere Reife und 73,3 \% verfügten über die Hochschulreife bzw. hatten einen akademischen Bildungsabschluss.

Befragt nach den der Therapie zugrunde liegenden Diagnosen (Mehrfachnennungen möglich), berichteten die meisten der Studienteilnehmer über eine Diagnose der Kategorie depressive Störungen (72,7\%). Angststörungen wurden am zweithäufigsten genannt (35,2\%), gefolgt von Traumafolgestörungen $(27,3 \%)$, PKS (24,8\%) und Essstörungen (12,7\%). Eine Person $(0,6 \%)$ benannte Schizophrenie als Therapieanlass. Im Mittel lag die Anzahl der berichteten Diagnosen bei $M=1,73$ $(S D=1,14)$. Die Therapien wurden in $62,4 \%$ der Fälle von weiblichen Therapeuten durchgeführt. Die mittlere Therapiedauer betrug $M=17,1$ Monate ( $S D=17,33$ Monate). Das Therapieende lag $M=6,36$ Jahre ( $S D=7,42$ Jahre) zurück. 41,2\% der Befragungsteilnehmer geben an, dass schon einmal ein Therapieabbruch bzw. ein Therapeutenwechsel stattgefunden hat. Weiter- 
- Tab. 1 Charakteristika der Behandlung.

\begin{tabular}{|l|c|}
\hline & $\begin{array}{l}\text { Häufigkeit } \\
\text { (in \%) }\end{array}$ \\
\hline Behandlungssetting & \\
\hline ambulant & 80,6 \\
\hline stationär & 29,7 \\
\hline tagesklinisch & 10,9 \\
\hline Behandlungsmodalität & \\
\hline Einzeltherapie & 77,6 \\
\hline Gruppentherapie & 28,5 \\
\hline Familientherapie & 0,6 \\
\hline Theoretische Ausrichtung & \\
\hline Verhaltenstherapie & 37,0 \\
\hline tiefenpsychologisch fundierte Psychotherapie & 24,2 \\
\hline Psychoanalyse & 9,7 \\
\hline systemische Therapie & 1,2 \\
\hline Gestalttherapie & 1,2 \\
\hline sonstige & 14,5 \\
\hline keine Angabe & 12,2 \\
\hline Berufsgruppe des Therapeuten & 10,9 \\
\hline psychologischer Psychotherapeut & 27,9 \\
\hline Facharzt für Psychiatrie und Psychotherapie & 8,5 \\
\hline Facharzt Psychosomatische Medizin \& Psycho- & \\
\hline therapie & \\
\hline keine Angabe & \\
\hline Anmerküglich des Behandlungssettings und der Behandlungsmoda- \\
\hline Märachnennungen möglich
\end{tabular}

führende Informationen zu den Charakteristika der Therapie und des therapeutischen Settings sind $\triangleright$ Tab. 1 zu entnehmen.

\section{Deskriptive Analysen}

Die durchschnittliche Anzahl der erlebten Ereignisse pro Patient über alle 12 Kategorien hinweg betrug $M=16,21$ (SD= 10,99). Die mittlere resultierende Belastung lag bei $M=2,06$ $(S D=0,9)$,

In > Tab. 2 sind die Auftretenshäufigkeit von Kunstfehlern gemittelt über die Anzahl der Items einer jeweiligen Kategorie sowie die mittlere berichtete Belastung je Kategorie dargestellt.

Die Analyse auf Einzelitemebene, unabhängig von der Beschwerdekategorie, ergab, dass den folgenden 3 Items am häufigsten zugestimmt wurde: „Ich hatte das Gefühl, ich mache keine Fortschritte in der Therapie.“ (72,7\% Zustimmung; Belastung: $M=2,75$; Kategorie: Mangelhafte Diagnostik/Technikanwendung); „Ich hatte das Gefühl, mit meinem Therapeuten/
- Tab. 2 Häufigkeit des Auftretens von Kunstfehlern entsprechend der zwölf Beschwerdekategorien gemittelt über die Anzahl der Items der jeweiligen Kategorie (Zahl in Klammern) und mittlere berichtete Belastung je Kategorie.

\begin{tabular}{|c|c|c|c|}
\hline & \multirow{2}{*}{$\begin{array}{l}\text { Auftretens- } \\
\text { häufigkeit } \\
\text { (in \%) }\end{array}$} & \multicolumn{2}{|c|}{ Belastung } \\
\hline & & $M$ & $S D$ \\
\hline Sexuelle GrÜ/GrV (9) & 6,01 & 3,40 & 0,60 \\
\hline Soziale GrÜ/GrV (9) & 9,81 & 2,51 & 1,11 \\
\hline Befangenheit (3) & 10,30 & 2,26 & 0,69 \\
\hline $\begin{array}{l}\text { Verletzung therapeutischer } \\
\text { Basisvariablen (10) }\end{array}$ & 40,91 & 2,83 & 0,40 \\
\hline $\begin{array}{l}\text { Mangelhafte Diagnostik/ } \\
\text { Technikanwendung (8) }\end{array}$ & 44,31 & 2,70 & 0,19 \\
\hline $\begin{array}{l}\text { Verstoß gegen therapeu- } \\
\text { tische Pflichten (6) }\end{array}$ & 15,55 & 2,86 & 0,52 \\
\hline $\begin{array}{l}\text { Ökonomischer/finanzieller } \\
\text { Missbrauch (6) }\end{array}$ & 3,11 & 2,07 & 1,15 \\
\hline $\begin{array}{l}\text { Therapieabbruch durch } \\
\text { Therapeuten (3) }\end{array}$ & 31,90 & 3,08 & 0,33 \\
\hline $\begin{array}{l}\text { Mangelhafte Rahmen- } \\
\text { bedingungen (7) }\end{array}$ & 11,77 & 1,95 & 1,18 \\
\hline Sonstiges (8) & 20,44 & 2,72 & 0,84 \\
\hline $\begin{array}{l}\text { GrÜ/GrV im Rahmen } \\
\text { stationärer Therapie (6) }\end{array}$ & 32,65 & 3,03 & 0,27 \\
\hline $\begin{array}{l}\text { GrÜ/GrV im Rahmen von } \\
\text { Gruppetherapie (9) }\end{array}$ & 28,34 & 2,46 & 0,60 \\
\hline
\end{tabular}

meiner Therapeutin nicht über alles reden zu können." (65,5\% Zustimmung; $M=2,43$; Verletzung therapeut. Basisvariablen) und „Ich fühlte mich durch meinen Therapeuten/meine Therapeutin missverstanden oder nicht ernst genommen.“ (63,6\% Zustimmung; $M=2,90$; Verletzung therapeut. Basisvariablen).

Innerhalb jeder Kategorie gab es starke Unterschiede hinsichtlich der Zustimmung zu einzelnen Items, was am Beispiel der Kategorie „Verletzung therapeutischer Basisvariablen“ verdeutlicht wird. Das Item „Ich glaube, mein Therapeut/meine Therapeutin hat mein Vertrauen missbraucht, um eigene Interessen zu verfolgen.“, wurde von 17,0\% der Befragten bejaht. Die größte Zustimmung (65,5\%) erzielte in dieser Kategorie das Item „Ich hatte das Gefühl, mit meinem Therapeuten/meiner Therapeutin nicht über alles reden zu können.".

Die 3 Items, bei denen die höchsten Belastungswerte benannt wurden, sind „Die Therapie fand wiederholt außerhalb der Praxisräume statt (z.B. Therapiesitzungen im Restaurant; beim Therapeuten/bei der Therapeutin zu Hause-Ausnahmen bilden z.B. Konfrontationsübungen im Fahrstuhl, in der Straßenbahn, im Kaufhaus).“ (3\% Zustimmung; $M=4,00$; Mangelhafte Rahmenbedingungen), „Mein Therapeut/meine Therapeutin hat mich körperlich angegriffen." (3\% Zustimmung; $M=$ 4,00; Sonstiges) und „Es kam während der Therapie zu sexuellen Handlungen zwischen mir und meinem Therapeuten/mei- 
ner Therapeutin (z. B. Berührung der Geschlechtsorgane, Geschlechtsverkehr, Oralverkehr)." (1,8\% Zustimmung; $M=4,00$; Sexuelle GrÜ/GrV).

Auch die Werte der mittleren Belastung schwankten innerhalb einer jeden Kategorie teils recht stark. In der Kategorie „Sexuelle GrÜ/GrV“ erzielte die größte Zustimmung das Item „Mein Therapeut/meine Therapeutin hat mir schmeichelhafte Komplimente gemacht.“ (15,8\%), es ging aber mit der geringsten Belastung einher $(M=1,08)$. Items wie „Es kam nach Beendigung der Therapie zu sexuellen Handlungen zwischen mir und meinem Therapeut/meiner Therapeutin (z. B. Berührung der Geschlechtsorgane, Geschlechtsverkehr, Oralverkehr).“ (0,6\% Zustimmung) oder „Ich hatte eine Beziehung mit meinem Therapeuten/meiner Therapeutin.“ (1,2\% Zustimmung) wurden seltener bejaht, gingen aber immer mit maximaler Belastung $(M=4)$ einher.

Insgesamt gaben 35,2\% der Studienteilnehmer an, zum Zeitpunkt der Befragung noch immer unter den Erfahrungen ihrer psychotherapeutischen Behandlung zu leiden.

\section{Inferenzstatistische Analysen}

In den linearen Regressionen erwies sich lediglich die Anzahl der Diagnosen des Patienten als prädiktiv für die Anzahl der berichteten Kunstfehler. Patienten mit mehr Diagnosen berichteten häufiger von Kunstfehlern (beta $=0,30 ; p=0,00$ ). Bei der Analyse der Störungsbilder zeigte sich vor allem ein Zusammenhang für PKS und Essstörungen. Patienten mit diesen Diagnosen berichteten häufiger von GrV und GrÜ (PKS: beta=0,22; $p=0,01$; Essstörungen: beta $=0,18 ; p=0,02$ ). Bezüglich der erlebten Belastung erwies sich keine der untersuchten Variablen als prädiktiv.

\section{Diskussion}

Die kontinuierliche und systematische Reflektion sowie Bewertung des therapeutischen Handelns in der Versorgungspraxis ist im Hinblick auf die Qualitätssicherung in der Psychotherapie notwendig und von hoher klinischer Relevanz [16], wobei besonders auch auf unerwünschte Entwicklungen während oder infolge einer Psychotherapie zu achten ist. Mit der Frage, inwieweit UE in der Person des Therapeuten und dessen Handeln begründet sind, beschäftigt sich die Forschung zu Kunstfehlern im therapeutischen Prozess - ein Feld, das lange Zeit wenig empirisch beforscht worden ist. Um das Auftreten von Kunstfehlern und deren Folgen aus Patientensicht systematisch und differenziert erfassen zu können, wurde ein neuer Fragebogen (IKKIP) entwickelt, dessen Einsatz in der einer Pilotierungsstudie mittels Online-Befragung erstmals getestet wurde.

Mit 78,2\% war der Großteil der Studienteilnehmer weiblich, was der Geschlechterverteilung in anderen Studien gleichkommt [4, 7, 17] und am wahrscheinlichsten auf das Inanspruchnahmeverhalten von Psychotherapie zurückgeführt werden kann. Zudem ist der überwiegende Anteil der Stichprobe entsprechend des erreichten Schul- bzw. Bildungsabschlusses als gut gebildet zu bezeichnen.

Im Mittel berichtete jeder Patient von etwa 16 Ereignissen im therapeutischen Kontakt, die nach dem Kategoriensystem
Kunstfehlern zuzuordnen sind und mit einer resultierenden Belastung mittelmäßiger Schwere beschrieben wurden. Dass 35,2\% der Studienteilnehmer angaben, zum Zeitpunkt der Studienteilnahme noch immer unter den Erfahrungen aus der Psychotherapie zu leiden, verdeutlicht die Nachhaltigkeit der Konsequenzen therapeutischen Fehlverhaltens.

Die Ergebnisse der Studie zeigen, dass im Mittel am häufigsten den Items der Kategorie „Mangelhafte Diagnostik/Technikanwendung“ zugestimmt wurde $(M=44,31 \%)$. Betrachtet man die Ergebnisse auf Einzelitemebene, dann wird deutlich, dass das Item „Ich hatte das Gefühl, ich mache keine Fortschritte in der Therapie." in dieser Kategorie die mit Abstand größte Zustimmung erhielt (72,7\%). Kritisch ist in diesem Zusammenhang zu hinterfragen, ob mit diesem Item tatsächlich ein Kunstfehler im Sinne eines therapeutischen Fehlverhaltens abgebildet wird oder Patienten damit einen im Rahmen einer Therapie durchaus häufig vorkommenden Punkt der Stagnation bekunden, der nicht zwangsläufig in der Person des Therapeuten und dessen Verhalten begründet sein muss. Berechnet man die mittlere Belastung dieser Kategorie folglich ohne jenes Item, sinkt der Belastungswert auf $M=40,25 \%$ und wäre somit geringer als der der Kategorie „Verletzung therapeutischer Basisvariablen“ $(M=40,91 \%)$. Letztere wurde in der Arbeit von Franke et al. [18] zur Analyse von Daten des Ethikvereins e. V. als diejenige Kategorie identifiziert, der die meisten berichteten Kunstfehler zugeordnet wurden (s. auch [12]).

Über $80 \%$ der Teilnehmer gaben an, psychotherapeutische Erfahrungen im ambulanten Setting gemacht zu haben. Im Zuge der zunehmend differenzierten Betrachtung negativer Effekte von Psychotherapie wurden in den vergangenen Jahren Fragebögen speziell für verschiedene Settings [19] und Patientenpopulationen [20] entwickelt. Zudem wurden verschiedene Inventare gezielt im Bereich ambulanter [17] oder stationärer Therapie [21] eingesetzt, um differenzierte Aussagen zu den Besonderheiten der jeweiligen Settings hinsichtlich negativer Therapieeffekte treffen zu können. Um diesen Entwicklungen Rechnung zu tragen, sind im IKKIP gesondert Items für den Bereich der stationären sowie für den der Gruppenpsychotherapie enthalten. Dass die Implementierung von speziell für diese beiden Settings konzipierten Items gerechtfertigt und durchaus wichtig ist, zeigen die Antworten der Teilnehmenden, die Erfahrungen in diesem Bereich bekundeten (Gruppe: 28,5\%; stationär: 29,7\%). Sowohl die mittlere Anzahl der Ereignisse als auch die damit einhergehende Belastung liegen bei diesen settingspezifischen Items verglichen mit den anderen Beschwerdekategorien im mittleren bis höheren Bereich und weisen somit auf Fehlentwicklungen im Sinne von GrÜ und GrV hin, die mit ernst zu nehmenden Belastungen für die Patienten einhergehen können.

Therapeutisches Fehlverhalten der Kategorie „Sexuelle GrÜ/ GrV“ wurde in der vorliegenden Studie immerhin im Mittel von $6,01 \%$ der Patienten berichtet. Diesbezüglich ist anzumerken, dass diese Kategorie sowohl Items zu verbalen sexuellen Grü und GrV als auch einige zum tatsächlichen Auftreten von sexuellen Handlungen während oder nach der Therapie beinhaltet, womit entsprechend des „slippery slope“-Musters verschiedene Stadien sexueller GrV bis zum sexuellen Missbrauch erfasst 
werden sollen (vgl. [9-11]). Unabhängig davon zeigte sich aber über alle Items hinweg, dass therapeutisches Fehlerverhalten in diesem Bereich mit der größten Belastung bei den Patienten einhergeht. Wenn es Therapeuten nicht gelingt, eigene persönliche Bedürfnisse und Interessen zugunsten des Fortgangs des psychotherapeutischen Prozesses zurückzustellen und sich somit nicht an das Abstinenzgebot halten, kann dies für Patienten mit schwerwiegenden Folgen und Schädigungen einhergehen, wie auch andere Arbeiten schlussfolgern [22]. In diesem Zusammenhang wird beispielsweise neben einer Verschlechterung der Ausgangssymptomatik auch das Auftreten neuer Beschwerden und Probleme wie sozialer Rückzug, Angst, Misstrauen, Traurigkeit, Depression und Aggression genannt [5, 23]. Obwohl im Allgemeinen belegt ist, dass nach einer ambulanten Psychotherapie die Zahl von Fehltagen im Sinne einer Arbeitsunfähigkeit im Mittel um 8 Tage geringer ist als vor der Behandlung [24], beschreibt Munz [25] eine zum Teil mehrere Jahre andauernde Arbeitsunfähigkeit von Patienten, bei denen es zu sexuellen GrV im Rahmen der Therapie gekommen ist.

Das IKKIP ist so konzipiert, dass es unabhängig vom Störungsbild der Befragten universell angewendet werden kann. Damit ist es mittels IKKIP grundsätzlich möglich, der Frage nachzugehen, ob sich im Hinblick auf das Erleben von Kunstfehlern und dem daraus resultierenden Belastungserleben unterschiedliche Befunde für verschiedene Störungsgruppen zeigen.

In der Pilotierungsstichprobe hatte der überwiegende Anteil der Studienteilnehmer (72,7\%) eine Diagnose aus dem Störungsspektrum der depressiven Störungen, doch waren auch andere Störungsbilder vertreten, sodass eine störungsheterogene Stichprobe resultierte, in der Komorbiditäten zudem nicht ausgeschlossen waren. Die Ergebnisse der inferenzstatistischen Analysen belegen, dass die Anzahl der Diagnosen das Auftreten von Kunstfehlern prädizierte, wobei mit steigender Diagnosezahl auch vermehrt GrÜ und GrV berichtet wurden. Hinsichtlich des Störungsbilds konnten die Diagnosen PSK und Essstörungen als Prädiktoren für das Erleben von Kunstfehlern identifiziert werden. Die Diagnose PSK ging bereits in der Studie von Ladwig et al. [7] mit einem erhöhten Erleben von negativen Effekten von Psychotherapie (gemessen mittels INEP) einher. Die Autoren diskutieren in dem Zusammenhang, ob dieser Befund mit dem Mangel an interpersonellen Fähigkeiten dieser Patientengruppe zusammenhängt, der womöglich auch im therapeutischen Kontext zu Problemen führen könnte, wodurch die Gefahr für subjektiv erlebte negative Effekte besteht.

Ob diese Befunde zur Rolle von Psychopathologie für das Auftreten bzw. Wahrnehmen von Kunstfehlern replizierbar sind und inwiefern auch andere Patientenmerkmale, wie zum Beispiel von Strauß et al. [26] untersucht, damit im Zusammenhang stehen, sollte in folgenden Studien genauer betrachtet werden.

\section{Limitationen und Ausblick}

Ausgehend von den Ergebnissen dieser Pilotierung sollen die Items des Fragebogens im nächsten Schritt kritisch überprüft und hinsichtlich des Verständnisses gegebenenfalls umformuliert werden.
43,5\% der Studienteilnehmer brachen die Beantwortung der Befragung vorzeitig ab bzw. füllten den Fragebogen nicht vollständig aus. Diese Schwundquote, die leicht über den Verlustraten anderer Online-Befragungen zu negativen Effekten von Psychotherapie liegt [7], könnte in der Länge des Inventars begründet sein. Ausgangspunkt für die Entwicklung des IKKIP war das Anliegen, das Repertoire möglicher Kunstfehler möglichst umfassend abzubilden, woraus die Gesamtitemzahl von 84 resultierte. Dadurch ist die Bearbeitungsdauer des IKKIP verglichen mit der anderer Fragenbögen deutlich länger und das Inventar in seiner jetzigen Form weniger ökonomisch. Entsprechend ist der Frage nachzugehen, ob einzelne Items gestrichen werden können, sodass eine Version resultiert, die das Konstrukt der Kunstfehler immer noch umfassend abbildet, die aber mit geringeren Abbruchraten einhergeht und eine größere Ökonomie aufweist. Dadurch könnte auch die Grundlage für den Einsatz des IKKIP in größeren Untersuchungen geschaffen werden.

Da die Stichprobenzusammensetzung auf Basis der Selbstselektion beruht, können aus den Ergebnissen keine Schlussfolgerungen im Hinblick auf die Häufigkeit des Auftretens von Kunstfehlern im Sinne der Repräsentativität getroffen werden. Folglich wäre es ratsam, eine Zufallsstichprobe [27] von Psychotherapiepatienten zu ihren Erfahrungen im therapeutischen Kontakt zu befragen, um aussagekräftigere Ergebnisse zu erzielen.

Peth et al. [28] zeigen, dass der Einsatz von Inventaren zu negativen Effekten auch in störungshomogenen Gruppen sinnvoll sein kann, um störungsspezifisch Schlussfolgerungen ziehen und daraus Empfehlungen für die gezielte Behandlung ableiten zu können. Entsprechend ist auch ein Einsatz des IKKIP in störungshomogenen Stichproben in Erwägung zu ziehen. Zudem ist im Zuge der personalisierten Psychotherapieforschung [29] die Erfassung negativer Effekte mehr in den Fokus zu rücken, um die Frage der differenziellen Wirkung von Psychotherapie allumfassend zu beleuchten, wozu neben der Wirkweise auch die Nebenwirkungen beforscht werden sollten.

Mittels IKKIP werden Kunstfehlerfolgen ausschließlich im Sinne der erlebten Belastung erhoben. Unklar bleibt, inwiefern die Befragten den Begriff der Belastung individuell definieren, wie sich diese äußerte und ob zudem auch andere Folgen für die Befragten resultierten.

Die Ergebnisse des IKKIP bilden die subjektive Sicht des Patienten auf den therapeutischen Prozess und das therapeutische Verhalten ab. Ausgehend von dieser Version des Fragebogens ist keine Information darüber vorhanden, wie der zugehörige Therapeut dieselbe Situation und sein eigenes Verhalten bewertet. Für diese Einschätzung ist entscheidend, wie der Therapeut GrV individuell definiert, diese wahrnimmt und welche Bereitschaft er hat, sein eigenes Handeln kritisch zu reflektieren. Mit dem Exploitation Index [30] existiert ein Inventar zur Erfassung grenzverletzender und grenzüberschreitender Verhaltensweisen, das primär Therapeuten adressiert, um sie im Sinne einer Selbstreflektion für problematische Verhaltensweisen zu sensibilisieren, wodurch bestenfalls eine Änderung des Verhaltens erzielt wird.

In diesem Sinne haben wir in Analogie aus der bestehenden Version des IKKIP eine Therapeutenversion entwickelt, die mo- 
mentan ebenfalls in einer anonymen Online-Befragung erstmals eingesetzt wird, um Aufschluss darüber zu erlangen, wie Behandler Grenzen in ihrer psychotherapeutischen Tätigkeit definieren und wie häufig Kunstfehler aus therapeutischer Sicht vorkommen, da auch dazu bislang nur sehr wenig bekannt ist.

Mit der Patienten- und der Therapeutenversion des IKKIP kann es nach deren Weiterentwicklung perspektivisch möglich sein, die beiden Versionen im Rahmen derselben Therapie einzusetzen und somit die individuellen Sichtweisen von Patient und Therapeut bezüglich des Auftretens von Kunstfehlern abzubilden und vergleichend gegenüberzustellen.

Mit Blick auf die praktische Relevanz scheint das IKKIP nicht zuletzt wegen seiner Länge jedoch weniger dazu geeignet zu sein, in laufenden Behandlungen regelmäßig die Patientensicht bzgl. des Auftretens von Kunstfehlern zu erheben. Vielmehr besteht mithilfe des Inventars die Möglichkeit, im Bereich der empirischen Forschung die Häufigkeit, Charakteristika und Korrelate von Grü und GrV zu untersuchen. Dies bietet die Grundlage zur Entwicklung von möglichen Interventionen aber auch Informations- und Schulungsmaterialien, um sowohl Patienten, aber besonders auch Therapeuten für dieses wichtige Thema zu sensibilisieren und professionelle Standards zu sichern. Mit einem umfassenden Bewusstsein und Wissen über Kunstfehler und deren Folgen, kann im Sinne der Prävention die Anzahl von GrV und GrÜ womöglich reduziert oder zumindest ein Beitrag dazu geleistet werden, diese zu identifizieren und mögliche resultierende Belastungen zu minimieren.

\section{KONSEQUENZEN FÜR KLINIK UND PRAXIS}

- Die kontinuierliche Reflektion sowie Bewertung des therapeutischen Handelns ist im Hinblick auf die Qualitätssicherung in der Psychotherapie notwendig und von hoher klinischer Relevanz.

- Auch Nebenwirkungen und unerwünschte Effekte einer Psychotherapie sowie Fehlentwicklungen in der therapeutischen Beziehung müssen genau beleuchtet werden.

- Mit dem Inventar zur Erfassung von Kunstfehlern und Kunstfehlerfolgen in der Psychotherapie ist es möglich, therapeutisches Fehlverhalten differenziert und systematisch aus Patientensicht zu erfassen sowie deren resultierende Belastung abzubilden.

Interessenkonflikt

Die Autoren geben an, dass kein Interessenkonflikt besteht.

\section{Literatur}

[1] Wampold BE, Imel ZA, Flückiger C, eds. Die Psychotherapie-Debatte. Was Psychotherapie wirksam macht. Göttingen: Hogrefe; 2018

[2] Haupt M-L, Linden M, Strauß B. Definition und Klassifikation von Psychotherapie-Nebenwirkungen. In: Linden M, Strauß B, eds. Risiken und Nebenwirkungen von Psychotherapie. Berlin: Medizinisch Wissenschaftliche Verlagsgesellschaft; 2018: 1-14

[3] Linden M, Strauß B, Scholten S et al. Definition und Entscheidungsschritte in der Bestimmung und Erfassung von Nebenwirkungen von Psychotherapie. Psychother Psych Med 2018; 68: 377-382

[4] Abeling B, Müller A, Stephan M et al. Negative Effekte von Psychotherapie: Häufigkeit und Korrelate in einer klinischen Stichprobe. Psychother Psych Med 2018; 68: 428-436

[5] Rozental A, Kottorp A, Boettcher J et al. Negative effects of psychological treatments: An exploratory factor analysis of the negative effects questionnaire for monitoring and reporting adverse and unwanted events. PLoS ONE 2016; 11: doi:10.1371/journal.pone. 0157503

[6] Boyle K, Lutz W. Behandlungsintegrität in der Psychotherapieforschung. Psychother Psych Med 2019; 69: 301-302

[7] Ladwig I, Rief W, Nestoriuc Y. Welche Risiken und Nebenwirkungen hat Psychotherapie? - Entwicklung des Inventars zur Erfassung negativer Effekte von Psychotherapie (INEP). Verhaltenstherapie 2014; 24: 252-263

[8] Scholten S, Velten J, Kintscher M et al. Beschwerden über Psychotherapie und ihre Auswirkungen auf Patienten und Patientinnen. Psychother Psych Med 2018; 68: 423-427

[9] Schleu A. Empathieversagen und Grenzverletzungen in der Psychotherapie von komplex traumatisierten Patienten. Trauma \& Gewalt 2018; 12: 308-321

[10] Schleu A. Das Spektrum von Grenz- und Abstinenzverletzungen in der Psychotherapie. Sexuelle und andere Grenzverletzungen. Psychotherapeut; 2019: im Druck

[11] Kontny L, Strauß B, Schleu A et al. Von der Komplexität doppelter Beziehungsangebote. Eine inhaltsanalytische Untersuchung sozialer Grenzverletzungen in der Psychotherapie. Psychotherapeut; 2019: im Druck

[12] Stuhler H, Strauß B, Schleu A et al. Von schleichenden Grenzverletzungen zu sexuellem Missbrauch. Eine systematische Auswertung von Patientenberichten. Psychotherapeut; 2019: im Druck

[13] Kaczmarek S, Passmann K, Cappel R et al. Wenn Psychotherapie schadet... Systematik von Beschwerden über psychotherapeutische Behandlungen. Psychotherapeut 2012; 57: 402-409

[14] Schleu A, Hillebrand V. Grenzverletzungen in der Psychotherapie und ihre Prävention. In: Egle U, Joraschky P, Lampe A et al., eds. Missbrauch, Misshandlung, Vernachlässigung. Stuttgart: Schattauer; 2016: 764-794

[15] Schwartze D, Strauss B. Zur Definition und Erfassung von Kunstfehlern und Kunstfehlerfolgen in der Psychotherapie. Trauma \& Gewalt 2018; 12: 296-307

[16] Rubel JA, Zimmermann D, Müller V et al. Qualitätssicherung in der Psychotherapie. Psychother Psych Med 2017; 67: 436-448

[17] Grüneberger A, Einsle F, Hoyer J. Subjektiv erlebte Nebenwirkungen ambulanter Verhaltenstherapie: Zusammenhänge mit Patientenmerkmalen, Therapeutenmerkmalen und der Therapiebeziehung. Psychother Psych Med 2017; 67: 338-344

[18] Franke P, Schleu A, Hillebrand V et al. Beschwerden über Fehlverhalten in der Psychotherapie, Teil 1: Quantitative und qualitative Analyse der Dokumentation des Vereins Ethik in der Psychotherapie 20062015. Psychotherapeut 2016; 61: 507-515

[19] Strauß B, Drobinskaya A. Erste Erfahrungen mit dem „Fragebogen zu Nebenwirkungen in der Gruppentherapie und unerwünschten Gruppenerfahrungen“ (NUGE 24). Psychother Psych Med 2018; 68: 437442

[20] Bieda A, Pflug V, Scholten S et al. Unerwünschte Nebenwirkungen in der Kinder- und Jugendlichenpsychotherapie - Eine Einführung und Empfehlungen. Psychother Psych Med 2018; 68: 383-390 
[21] Brakemeier EL, Herzog P, Radtke M et al. CBASP als stationäres Behandlungskonzept der therapieresistenten chronischen Depression: Eine Pilotstudie zum Zusammenhang von Nebenwirkungen und Therapieerfolg. Psychother Psych Med 2018; 68: 399-407

[22] Strauß B, Schwartze D, Freyberger H. Traumatische Nebenwirkungen in der Psychotherapie. Trauma \& Gewalt 2018; 12: 284-292

[23] Eichenberg C, Dorniak J, Fische G. Sexuelle Übergriffe in therapeutischen Beziehungen: Risikofaktoren, Folgen und rechtliche Schritte. Psychother Psych Med 2009; 59: 337-344

[24] Epping J, de Zwaan M, Geyer S. Gesünder nach der Psychotherapie? Psychother Psych Med 2018; 68: 337-345

[25] Munz D. Abstinenz als ethische Grundhaltung in der Psychotherapie. Vortrag auf der Fachtagung der Landespsychotherapeutenkammer Baden-Württemberg zu "Verantwortung in der Psychotherapie: Abstinenz aus fachlicher und juristischer Sicht", 2010: Im Internet: http://www.lpk-bw.de/archiv/news2010/pdf/100711_abstinenz_ als_ethische_grundhaltung.pdf (Zugriff: 01.10.2019). (Bericht über die Tagung unter dem Titel „Verantwortung in der Psychotherapie: Abstinenz aus fachlicher und juristischer Sicht - Fachtagung der LPK BW« im Psychotherapeutenjournal, 9, 294-296.)
[26] Strauß B, Grass N, Ritter V et al. Negative Indikatoren im psychotherapeutischen Prozess: Häufigkeit und Zusammenhang mit Bindungsmerkmalen und Therapieerfolg bei Patienten mit sozialer Angststörung. Psychother Psych Med 2018; 68: 408-416

[27] Jacobsen J, Richter D. Gibt es repräsentative Umfragen? Psychother Psych Med 2019; 69: 203-204

[28] Peth J, Jelinek L, Nestoriuc Y et al. Unerwünschte Effekte von Psychotherapie bei depressiven Patienten - Erste Anwendung der Positive and Negative Effects of Psychotherapy Scale (PANEPS). Psychother Psych Med 2018; 68: 391-398

[29] Lutz W, Wucherpfennig F. Was bedeutet Personalized Medicine und Personalized Mental Health für die Psychotherapie und Psychotherapieforschung? Oder warum die Psychotherapieforschung in Deutschland ausgebaut werden sollte/könnte/müsste? (Teil 3). Psychother Psych Med 2017; 67: 227-230

[30] Epstein RS, Simon RI. The Exploitation Index: An early warning indicator of boundary violations in psychotherapy. Bull Menninger Clin 1990; 54: 450-465 